\title{
Marine life science and technology (MLST): a new journal highlighting all aspects of marine biology and biotechnology research
}

\author{
Hongan Long ${ }^{1} \cdot$ Xiao-Hua Zhang ${ }^{2,3}$
}

Received: 9 October 2019 / Accepted: 5 November 2019 / Published online: 2 December 2019

(C) Ocean University of China 2019

The majestic nature of the oceans and their charismatic flora and fauna clearly justify the study of marine life, especially to the general public. Consequently, the exploration of marine biota has provided both insights and highlighted challenges, leading to the appreciation that marine biology and its supporting technology must be at the forefront of $21 \mathrm{st}$ century science. Still, this field is not progressing as fast or in as much detail as might be expected, or needed. Possibly the most notable gap is the lack of academic journals that cover cross-disciplinary topics.

Marine sciences are facing a growing number of complex challenges that researchers are currently unable to solve through studies in any single discipline. We suggest that the solution is to foster synergies arising from crossdisciplinary studies. For example, many marine drugs and lead compounds have been discovered, directly or indirectly, through studies on the exploitation of marine bioresources that employ genetic techniques and evaluate chemical ecology. Likewise, studies on the community structure of marine plankton and their ecological functions have facilitated our understanding of marine environmental sciences. Our new journal provides a venue for such high-impact, cross-disciplinary studies.

Specifically, Marine Science and Technology (MLST) now offers a platform that introduces discoveries and theories associated with marine organisms, fisheries science, marine drugs, bioproducts, and bioresources, with an

\section{Edited by Chengchao Chen.}

Xiao-Hua Zhang

xhzhang@ouc.edu.cn

1 Institute of Evolution and Marine Biodiversity, KLMME, Ocean University of China, Qingdao 266003, China

2 College of Marine Life Sciences, Ocean University of China, 5 Yushan Road, Qingdao 266003, China

3 Laboratory for Marine Ecology and Environmental Science, Qingdao National Laboratory for Marine Science and Technology, Qingdao 266071, China emphasis, but not exclusively, on biological technology and biological oceanography. It is co-sponsored by two leading institutions in marine science studies- the Ocean University of China and the Pilot National Laboratory for Marine Science and Technology, China- and is published by SpringerNature Press. The sponsors will cover all fees including those supporting Open-Access for articles published before 2022. To ensure the success of this comprehensive journal we have involved more than 80 editors, all of whom are active researchers (nearly two-thirds are from outside of mainland China) and three full-time professional editors.

To illustrate our scope, the first issue features eight review articles and two research articles that have been contributed by our editors; below, we introduce these.

Epigenetic research focuses on heritable changes beyond DNA, a phenomenon that will influence all marine life. The model microbial eukaryote Tetrahymena has facilitated numerous studies and benefited many fields. In our first paper, Cheng et al. (2019) summarize their progress in understanding epigenetic mechanisms using Tetrahymena. This review reports their efforts to generate a high-resolution DNA $\mathrm{N}^{6}$-methyladenine (6 $\left.\mathrm{mA}\right)$ map, evaluate the determinants for nucleosome distribution, demonstrate the roles of RNAi-dependent Polycomb repression pathways on transposable elements, and discover the functions of histone methylation in replication elongation. Admittedly, Tetrahymena is a freshwater species, but we recognize that epigenetics studies in non-marine model species not only shed light into understanding conserved pathways in eukaryotes but also provide technical guidance for future marine epigenetics studies.

High-throughput genome-sequencing techniques continue to revolutionize studies on genomics and metagenomics of various marine organisms, and per-sample costs have been decreasing dramatically. However, the total budget for large-scale genome-sequencing sets significant research barriers. In our second paper Li et al. (2019) present two cost-effective strategies for genomics 
experiments: (1) a miniaturized DNA library preparation protocol and (2) a laboratory instrument-making system driven by $3 \mathrm{D}$-printing-technology. The authors share their original protocols and 3D models and demonstrate both approaches greatly reduce the cost of building a genomics laboratory without compromising the accuracy of the data.

New gene cloning methods will speed up genetic studies of many organisms. Species in the microalgal genus Nannochloropsis are widely used as models for theoretical and applied studies. In our third paper, Yang et al. (2019) introduce methods for de novo cloning the genes of fatty-acidrich microalga Nannochloropsis oceanica and for creating diverse genetic variations in its genome, specifically for the biotechnological applications of marine microalgae.

Morphogenesis is the process of pattern and structure formation during development. Marine animals show highly diverse morphogenesis processes as a result of their long evolution history in marine environments. Currently, most of our understanding of the molecular basis of morphogenesis has been obtained from the terrestrial model organisms; these are unlikely to explain the extensive morphological diversity that has arisen in the oceans, an environment that imposes very different evolutionary pressures. Marine taxa, therefore, provide new opportunities for developmental biologists to explore molecular machineries that control the development in a broader context. In our fourth paper, Lv et al. (2019) review the recent advancements of morphogenesis of three marine model animals (ascidians, sea urchins, sea anemones) at molecular, cellular, and tissue levels. This review focuses on the common mechanisms under diverse morphogenesis process, such as polarity establishment, tissue invagination, and tissue elongation. Furthermore, the review raises critical, unsolved morphological questions in the development of marine invertebrates.

Infectious diseases, especially those attributed to microorganisms, remain a major constraint in mariculture production. Diagnosis is the prerequisite for effective prevention and control of infectious diseases. In our fifth paper, to facilitate the management of fish diseases, Austin (2019) reviews the methodologies involved in the diagnosis of bacterial fish diseases, including traditional culture-dependent (culturing and phenotyping) techniques, serological systems (e.g., ELISA kits), and culture-independent (molecular biology) approaches.

Aquafeeds are constantly being improved. Efforts have been made to replace marine fish meal in aquafeeds with alternative protein sources due to the limited supply and high prices of fishmeal. However, fishmeal replacement can lead to inferior growth and health status. In our sixth paper, Liu et al. (2019a) address nutritional aspects of aquafeeds and describe amino acids controlling cell growth and metabolism through two amino acids sensing pathways, target of rapamycin complex 1 (TORC1), and general control nonderepressible 2 (GCN2) signaling pathways.

Marine metabolites are a reservoir for discovering potent bioactive molecules with diverse structures. The secondary metabolites from marine invertebrates and their symbiotic microorganisms represent a promising and valuable source for drug discovery. In our seventh paper, Liu et al. (2019b) provide a comprehensive introduction to marine natural products research. Multiple strategies and methods have been applied to find novel marine natural products with diverse structures and promising activities, including OSMAC (one strain many compounds), chemical epigenetic manipulation, co-culture, structural modification, and chemical synthesis.

Fertilizers from marine waste products are a potentially valuable resource. However, breaking down some marine products, such as chitin can be problematic. In our eighth paper, Fu et al. (2019) introduce an eco-friendly method for producing chitooligosaccharides, with different degrees of deacetylation (DDAs) from waste shrimp shells, and their effects on the germination of wheat seeds. This is a step towards making new natural fertilizers, as traditional methods for producing chitosan and their derivatives lead to environmental pollution and loss of nutrients.

Cosmeceutical ingredients that protect human skin from damage by UV irradiation are commercially valuable. Marine algae are rich in compounds that possess various bioactivities including UV protection. In our ninth paper, Wang et al. (2019) evaluate the antioxidant activities and UV-protective effect of sulfated polysaccharides, derived from the celluclast-assisted extract of a brown alga Hizikia fusiforme. They indicate that these polysaccharides significantly reduce UVB-induced photo-damage in in vitro and in vivo models and can be used as a potential natural cosmeceutical ingredient.

Marine microorganisms are major drivers of many biogeochemical processes and exert crucial roles in maintaining ecosystem and climate stability. With the development of high-throughput sequencing technology in the last $2 \mathrm{dec}-$ ades, vast amounts of data on microbial community composition have been obtained. Extensive mining of these data is providing new insights into marine microbial ecology. In our tenth paper, Liu et al. (2019c) summarize wide-ranging theoretical and practical findings on microbial community assembly mechanisms, co-occurrence patterns, activities, and functional predictions.

The future of MLST In summary, the 10 contributed articles in this first issue reflect, but in no way exhaust, the broad potential scope of MLST. These papers hopefully serve to stimulate further contributions from the marine science community. With our international editorial board and strong support from the academic sponsors and SpringerNature, we firmly set up our mind to grow MLST into a new, 
respected platform for high-impact and cross-disciplinary studies on marine biology and biotechnology.

\section{References}

Austin B (2019) Methods for the diagnosis of bacterial fish diseases. Mar Life Sci Technol 1:41-49

Cheng T, Wang Y, Huang J, Chen X, Zhao X, Gao S, Song WB (2019) Our recent progress in epigenetic research using the model ciliate, Tetrahymena thermophila. Mar Life Sci Technol 1:4-14

Fu X, Zhu L, Li L, Zhang T, Li M, Mou H (2019) Eco-friendly preparation of chitooligosaccharides with different degrees of deacetylation from shrimp shell waste and their effects on the germination of wheat seeds. Mar Life Sci Technol 1:96-104

Li H, Wu K, Ruan C, Pan J, Wang Y, Long H (2019) Cost-reduction strategies in massive genomics experiments. Mar Life Sci Technol $1: 15-21$

Liu C, Wang X, Zhou H, Mai K, He G (2019a) Recent advances in amino acid sensing and new challenges for protein nutrition in aquaculture. Mar Life Sci Technol 1:50-59
Liu L, Zheng Y, Shao C, Wang C (2019b) Metabolites from marine invertebrates and their symbiotic microorganisms: molecular diversity discovery, mining and application. Mar Life Sci Technol 1:60-95

Liu J, Meng Z, Liu X, Zhang X-H (2019c) Microbial assembly, interaction, functioning, activity and diversification: a review derived from community compositional data. Mar Life Sci Technol $1: 113-129$

Lv Z, Lu Q, Dong B (2019) Morphogenesis: a focus on marine invertebrates. Mar Life Sci Technol 1:28-40

Wang L, Oh JY, Yang H-W, Kim HS, Jeon Y-J (2019) Protective effect of sulfated polysaccharides from a Celluclast-assisted extract of Hizikia fusiforme against ultraviolet B-induced photoaging in vitro in human keratinocytes and in vivo in zebrafish. Mar Life Sci Technol 1:105-112

Yang G, Zhang Z, Liu H, Guo L (2019) An investigation of the possible methods and potential benefits of de novo cloning of Nannochloropsis oceanica genes. Mar Life Sci Technol 1:22-27 